\title{
A STRONG MAXIMUM PRINCIPLE FOR QUASILINEAR PARABOLIC DIFFERENTIAL INEQUALITIES
}

\author{
O. ARENA $^{1}$
}

Abstract. A maximum principle for $C^{1}$ solutions of quasilinear parabolic differential inequalities which retains the strong conclusion of Nirenberg's well-known result [2] is established.

The case of strongly differentiable solutions rather than of class $C^{1}$ is also discussed.

1. Introduction. Statement of results. Let $Q$ be a domain in an $(n+1)$ dimensional space $(x, t) \equiv\left(x_{1}, \cdots, x_{n}, t\right)$.

In [2], L. Nirenberg derived a strong maximum principle for second order parabolic operators in $Q$ with continuous coefficients. This result, as it is pointed out in [2], can be carried over to nonlinear parabolic equations of the form

$$
\Phi\left(x, t, u, D u, D^{2} u\right)=u_{t},
$$

where $D u=\left(\partial u / \partial x_{1}, \cdots, \partial u / \partial x_{n}\right)$ denotes the spatial gradient and $D^{2} u$ the Hessian matrix of the dependent variable $u=u(x, t)$. Moreover it is assumed that the function $u$ has continuous first and second derivatives with respect to the $x_{i}$ and continuous first derivative with respect to $t$.

The purpose of this paper is to find a maximum principle which applies to $C^{1}$ solutions in $Q$ of parabolic differential inequalities and still has the strong conclusion of Nirenberg's result.

In this way we extend to a time-dependent situation an interesting maximum principle for $C^{1}$ solutions of elliptic differential inequalities recently established by J. Serrin in [3], that retains the strong conclusion of the well-known Hopf result [1].

We shall consider the second order quasilinear partial differential inequalities

$$
\begin{array}{r}
\operatorname{div} A(x, t, u, D u)-B(x, t, u, D u) \geqq u_{t}, \\
\operatorname{div} A(x, t, v, D v)-B(x, t, v, D v) \leqq v_{t},
\end{array}
$$

Received by the editors February 16, 1971 and, in revised form, July 21, 1971.

AMS 1969 subject classifications. Primary 3562.

Key words and phrases. Parabolic equations, quasilinear equations, strong maximum principle.

${ }^{1}$ This research was supported by a grant from the Consiglio Nazionale delle Ricerche (Italy) and was done at the School of Mathematics, University of Minnesota.

The author would like to thank Professor D. G. Aronson for several helpful discussions.

(c) American Mathematical Society 1972 
where $A=\left(A_{1}, \cdots, A_{n}\right)$ is a given vector function of $(x, t, u, D u)$ and $B$ is a given scalar function of the same variables. By div $A(x, t, u, D u)$ we mean the divergence, with respect to the variable $x$, of $A(x, t, u(x, t), D u(x, t))$.

As to the structure of the functions $A(x, t, u, p)$ and $B(x, t, u, p)$ we shall assume the following:

(i) the function $A(x, t, u, p)$ is differentiable with respect to the variables $u$ and $p$;

(ii) the function $B(x, t, u, p)$ is Lipschitz continuous in the variables $u$ and p.

Let $Q_{\tau}=Q \cap\{t=\tau\}$. Since for the main result we assume only that the functions $u$ and $v$ are of class $C^{1}$ in $Q$, we need to interpret (1) in the weak form:

$$
\begin{gathered}
\int_{Q}\left\{\phi u_{t}+D \phi \cdot A(x, t, u, D u)+\phi B(x, t, u, D u)\right\} d x d t \leqq 0, \\
\int_{Q}\left\{\phi v_{t}+D \phi \cdot A(x, t, v, D v)+\phi B(x, t, v, D v)\right\} d x d t \geqq 0,
\end{gathered}
$$

valid for all nonnegative $\phi \in C^{1}(Q)$, having compact support in $Q_{t}$, for each $t$.

To state our result it is convenient, for each point $\left(x_{0}, t_{0}\right) \in Q$, to denote by $S_{\left(x_{0}, t_{0}\right)}$ the set of all points $(x, t) \in Q$ which can be joined to $\left(x_{0}, t_{0}\right)$ by $a$ downward directed curve, with $\left(x_{0}, t_{0}\right)$ as initial point and $(x, t)$ as endpoint, namely by a curve given by the equations

$$
x_{i}=\varphi_{i}(t) \quad(i=1,2, \cdots, n),
$$

where $\varphi_{i}$ are of class $C^{0}$ on $\left[t, t_{0}\right], t<t_{0}$.

We shall prove the following:

TheORem 1. Let $u=u(x, t)$ and $v=v(x, t)$ be functions of class $C^{1}$ on $Q$. Suppose that $u=v$ at some point $\left(x_{0}, t_{0}\right) \in Q$ and that $u \leqq v$ in $S_{\left(x_{0}, t_{0}\right)}$. Assume further that $u$ and $v$ satisfy the respective differential inequalities (1) in $S_{\left(x_{0}, t_{0}\right)}$, where at least one of the matrices

$$
\partial A / \partial(D u) \text { or } \partial A / \partial(D v)
$$

is to be supposed positive definite. Then $u \equiv v$ in $S_{\left(x_{0}, t_{0}\right)}$.

REMARK 1. Let us introduce the notation

$$
K_{x_{0}}(\rho) \times\left(t_{0}-\tau \rho^{2}, t_{0}\right),
$$

where $K_{x_{0}}(\rho)$ stands for a cube of side $\rho$ and center $x_{0}$, to describe a spacetime rectangular neighborhood of $\left(x_{0}, t_{0}\right)$ on an $(n+1)$-dimensional space.

What essentially we shall prove is that, if the equality $u=v$ holds at a single point $\left(x_{0}, t_{0}\right)$, under the assumptions of the above theorem, then it 
holds everywhere on a rectangular neighborhood of $\left(x_{0}, t_{0}\right)$ of the form (3) contained in $Q$. From this fact then it shall follow that the equality $u=v$ holds in $S_{\left(x_{0}, t_{0}\right)}$.

Actually, as it is pointed out later after the proof of Theorem 1, the same result is valid under a weaker assumption on the function $A(x, t, u, p)$. More precisely we can assume that

(i') the function $A(x, t, u, p)$ is Lipschitz continuous in the variable $u$ and differentiable with respect to the variable $p$.

Next, to turn to solutions of (1) of class $W_{\text {loc }}^{1,2}$ in $Q$ we shall interpret (1) in the weak sense, that is in the sense that (2) are required to hold for all bounded nonnegative functions $\phi \in W^{1,2}(Q)$, having compact support in $Q_{t}$, for each $t .^{2}$

In this case if we assume that

(iii) the functions $A(x, t, u, p)$ and $B(x, t, u, p)$ are uniformly Lipschitz continuous with respect to the variables $u$ and $p$, we are able to prove the following:

THEOREM 2. Let $u=u(x, t)$ and $v=v(x, t)$ be continuous functions in $Q$, having strong derivatives with respect to $x$ and $t$ of class $L_{\mathrm{loc}}^{2}$. Suppose that $u=v$ at some point $\left(x_{0}, t_{0}\right) \in Q$ and that $u \leqq v$ in $S_{\left(x_{0}, t_{0}\right)}$. Assume further that $u$ and $v$ satisfy the respective differential inequalities (1) in $S_{\left(x_{0}, t_{0}\right)}$, where the matrix $\partial A / \partial p$ is to be supposed uniformly positive definite. Then $u \equiv v$ in $S_{\left(x_{0}, t_{0}\right)}$

Clearly the continuity assumption on $u$ and $v$ can be dropped provided that equalities and inequalities are considered in the almost everywhere sense, without essentially changing the proof.

The assumption on the matrix $\partial A / \partial p$ tells that the operator $\operatorname{div} A(x, t, u, D u)$ is uniformly elliptic, but it is worth emphasizing that such a condition is not required to state the main result.

To carry out the proofs we shall follow the outline`of Serrin's proof for the elliptic case [3]; here we shall invoke and suitably apply a remarkable time-dependent Harnack-type inequality proved by N. Trudinger in [4].

\section{Proof of results.}

Proof of Theorem 1. The main goal of the proof is to show that $u \equiv v$ on a rectangular neighborhood of $\left(x_{0}, t_{0}\right)$, as described in Remark 1 , contained in $Q$.

${ }^{2} W^{1,2}(Q)$ is the Sobolev space of all square integrable functions in $Q$, which have square integrable first derivatives with respect to $x$ and $t$. The derivatives are to be taken in the sense of distributions theory. $W^{1,2}(Q)$ is a Hilbert space under the norm $\|u\|=$ $\left(\int_{Q}\left(u^{2}+\sum_{1}^{n} u_{x_{i}}^{2}+u_{t}^{2}\right) d x d t\right)^{1 / 2}$. We define the local space $W_{1 \mathrm{oc}}^{1,2}(Q)=\{u(x, t): u, D u$, $\left.u_{i} \in L_{\text {loc }}^{2}(Q)\right\}$. 
To this end let $R$ denote the rectangular neighborhood of $\left(x_{0}, t_{0}\right)$,

$$
R=K_{x_{0}}(\rho) \times\left(t_{0}-\tau \rho^{2}, t_{0}\right)
$$

$\rho$ and $\tau$ being chosen so small that $R$ is contained in $Q$.

We notice that as a matter of fact in the inequalities (2) we may restrict consideration to nonnegative functions $\phi$ which vanish outside $R$ and correspondingly the integrations can take place over $R$. So, as can readily be seen by subtracting the first inequality of (2) from the second, the following important relation holds:

$$
\begin{aligned}
& \int_{R}\left\{\phi\left(v_{t}-u_{t}\right)+D \phi\right. {[A(x, t, v, D v)-A(x, t, u, D u)] } \\
&+\phi[B(x, t, v, D v)-B(x, t, u, D u)]\} d x d t \geqq 0
\end{aligned}
$$

for all nonnegative functions $\phi \in C^{1}(R)$, which vanish outside $R_{t}$, for each $t$ $\left(R_{\tau}=R \cap\{t=\tau\}\right)$.

The hypotheses (i) and (ii) yield in $R$

$$
\begin{aligned}
& |A(x, t, v, D v)-A(x, t, u, D u)| \leqq a|D w|+b|w|, \\
& |B(x, t, v, D v)-B(x, t, u, D u)| \leqq c|D w|+d|w|,
\end{aligned}
$$

where $w$ denotes the function difference $v-u$ and $a, b, c, d$ are suitable constants depending on the structure of the functions $A$ and $B$ as well as on bounds for $u, v, D u$ and $D v$ in $R$.

Let the matrix $\partial A / \partial(D u)$ be assumed positive definite (in the other case the proof is carried out similarly). Then, in view of the continuity of the matrix $\partial A / \partial(D u)$, there exists a positive constant $\lambda$ such that, in $R$, $\partial A / \partial(D u) \geqq \lambda$.

As in [3], we can easily show that

(6) $(D w) \cdot\{A(x, t, v, D v)-A(x, t, u, D u)\} \geqq \frac{\lambda}{4}|D w|^{2}-\left(\frac{2 b^{2}}{\lambda}+\frac{\lambda}{2}\right) w^{2}$

in $R=K_{x_{0}}(\rho) \times\left(t_{0}-\tau \rho^{2}, t_{0}\right)$ provided that $\rho$ and $\tau$ are sufficiently small.

Let us now consider the nonnegative function $w$ as a solution of the differential inequality (4). Clearly the relations (5) and (6) allow us to apply a time-dependent Harnack-type inequality due to N. Trudinger [4, Theorem 1.2].

We wish to do this in a suitable manner and precisely applying repeatedly Trudinger's result to couples of subrectangles of $R$ separated by successively smaller nonempty time intervals. For this purpose, let us consider the following, respectively increasing and decreasing, sequences $\left\{R_{v}^{*}\right\}$ and 
$\left\{R_{v}^{-}\right\}$of subrectangles of $R$ :

$$
\begin{aligned}
& R_{v}^{*}=K_{x_{0}}\left(\rho^{\prime \prime}\right) \times\left(t_{0}-\tau \rho^{2}, t_{0}-\frac{1}{v} \rho^{2}\right), \\
& R_{v}^{-}=K_{x_{0}}\left(\rho^{\prime}\right) \times\left(t_{0}-\frac{1}{v+1} \rho^{2}, t_{0}\right),
\end{aligned}
$$

where $0<\rho^{\prime}<\rho^{\prime \prime}<\rho$ and the integer $\nu$ is such that $\nu \geqq[1 / \tau]+1$.

Thus, in view of the aforementioned Harnack-type inequality, we have

$$
\int_{t_{0}-\tau \rho^{2}}^{t_{0}-\rho^{2} / \nu} \int_{K_{x_{0}}\left(\rho^{\prime \prime}\right)} w(x, t) d x d t \leqq C \rho^{n+2} \cdot \min _{R_{\bar{v}}} w(x, t),
$$

for $\nu=[1 / \tau]+1,[1 / \tau]+2, \cdots$, where $C$ depends on the structure and on the geometric constant $\rho^{\prime} / \rho, \nu$ and $\tau$.

On the other hand, since $w$ is nonnegative on $R$ and $w\left(x_{0}, t_{0}\right)=0$, the right-hand side of (7) is zero for any $v$ and therefore we get

$$
\int_{t_{0}-\tau \rho^{2}}^{t_{0}-\rho^{2} / v} \int_{K_{x_{0}}\left(\rho^{\prime \prime}\right)} w(x, t) d x d t \leqq 0,
$$

for $\nu=[1 / \tau]+1,[1 / \tau]+2, \cdots$.

Letting $\nu \rightarrow \infty$ then yields

$$
\int_{t_{0}-\tau \rho^{2}}^{t_{0}} \int_{K_{x_{0}}\left(\rho^{\prime \prime}\right)} w(x, t) d x d t \leqq 0 .
$$

Hence $w \equiv 0$, namely $u \equiv v$, in the rectangular neighborhood of $\left(x_{0}, t_{0}\right)$

$$
K_{x_{0}}\left(\rho^{\prime \prime}\right) \times\left(t_{0}-\tau \rho^{2}, t_{0}\right),
$$

with $\tau$ and $\rho$ determined as above and $0<\rho^{\prime \prime}<\rho$.

Now the conclusion of the theorem, which is that $u \equiv v$ in $S_{\left(x_{0}, t_{0}\right)}$, follows by a standard argument.

REMARK 2. The same result holds even if the function $A(x, t, u, p)$ is assumed to satisfy instead of the condition (i) the weaker one (i').

As a matter of fact, in this case the proof can be carried out the same as before, except that when we consider the difference $A(x, t, v, D v)$ $A(x, t, u, D u)$ it will be more convenient to write it as follows

$$
\begin{aligned}
& A(x, t, v, D v)-A(x, t, u, D u) \\
& \quad=[A(x, t, u, D v)-A(x, t, u, D u)]+[A(x, t, v, D v)-A(x, t, u, D v)]
\end{aligned}
$$

and then to estimate separately.

Proof of THEOREM 2. The argument is exactly the same as that of Theorem 1. As there, estimates (5) hold in the rectangular neighborhood 
$R$ of $\left(x_{0}, t_{0}\right)$. Furthermore, condition (6) is still valid in $R$; in order to check this we shall make use of the remark above and take into account the fact that the matrix $\partial A / \partial p$ is uniformly positive definite. Therefore, we may apply, suitably as in the proof of Theorem 1, Trudinger's result to the function $w=v-u$. This leads to finding a suitable rectangular neighborhood of $\left(x_{0}, t_{0}\right)$ where $w \equiv 0$. Again that is enough to complete the proof of the theorem.

\section{REFERENCES}

1. E. Hopf, Elementare Bemerkungen über die Lösungen partieller Differentialgleichungen zweiter Ordnung vom elliptischen Typus, S.-B. Preuss. Akad. Wiss. 19 (1927), 147152.

2. L. Nirenberg, $A$ strong maximum principle for parabolic equations, Comm. Pure Appl. Math. 6 (1953), 167-177. MR 14, 1089; MR 16, 1336.

3. J. Serrin, On the strong maximum principle for quasilinear second order differential inequalities, J. Functional Analysis 5 (1970), 184-193. MR 41 \#3966.

4. N. Trudinger, Pointwise estimates and quasilinear parabolic equations, Comm. Pure Appl. Math. 21 (1968), 205-226. MR 37 \#1758.

School of Mathematics, University of Minnesota, Minneapolis, Minnesot a 55455

Seminario Matematico, Università di Catania, 95129 Catania, Italy 